\title{
Xerostomia: An unseen consequence of statin use
}

\section{BACKGROUND}

Xerostomia is defined as dry mouth resulting from reduced or absen saliva flow. There are two types: one type is associated with severely reduced salivary secretion, while the other type involves normal salivary reduced salivary secretion, while the other type involves normal salivary
function yet reduced viscosity of saliva or reduced mucin concentration function yet reduced viscosity of saliva or reduced
within the saliva, a common finding in elderly people

Several studies have shown that xerostomia is the most common oral side-effect of cardiovascular medication. ${ }^{2}$ Statins are common cardiovascular drugs used to treat hypercholesterolemia.

Statins are known to have adverse side effects. The most severe one being rhabdomyoloysis which is also one of the most infrequent, followed by myalgia yet these effects seem to regress following discontinuation of treatment. $^{3}$

\section{CASE REPORT}

A 55-year-old Asian lady, non-smoker, and a teetotal with bilateral xanthelasma was commenced on simvastatin in August 2011 due to hypercholesterolemia and elevated lipoprotein a. She was initially started on nicotinic acid as it is the most effective treatment for elevated lipoprotein a however as it caused her adverse side effects it was stopped and she was subsequently commenced on $20 \mathrm{mg}$ simvastatin, increased to $40 \mathrm{mg}$ after 4 weeks. Unfortunately she started to develop oral dryness within a few weeks (see Fiqure 2). This was present throughout the day and night.

Owing to reduced salivary flow rate as a result of snoring and mouth breathing during sleep, nocturnal oral dryness is a normal entity, yet daybreathing during sleep, nocturnal oral dryness is a normal entity, yet day-
time oral dryness is a finding associated with systemic diseases such as time oral dryness is a finding associated with systemic diseases such as Sjogrens disease, diabetes, vitamins $A$ and $E$ deficiencies for which she was negative or pharmaceutical drug use, one of which are statins. She was temporarly changed from simvastatin to atorvastatin due to persistently high LDL cholesterol and the high risk of both CVD and PVD associated with high lipoprotein a. Atorvastatin was also stopped for 4 weeks and it substantially did help to minimize her xerostomia.

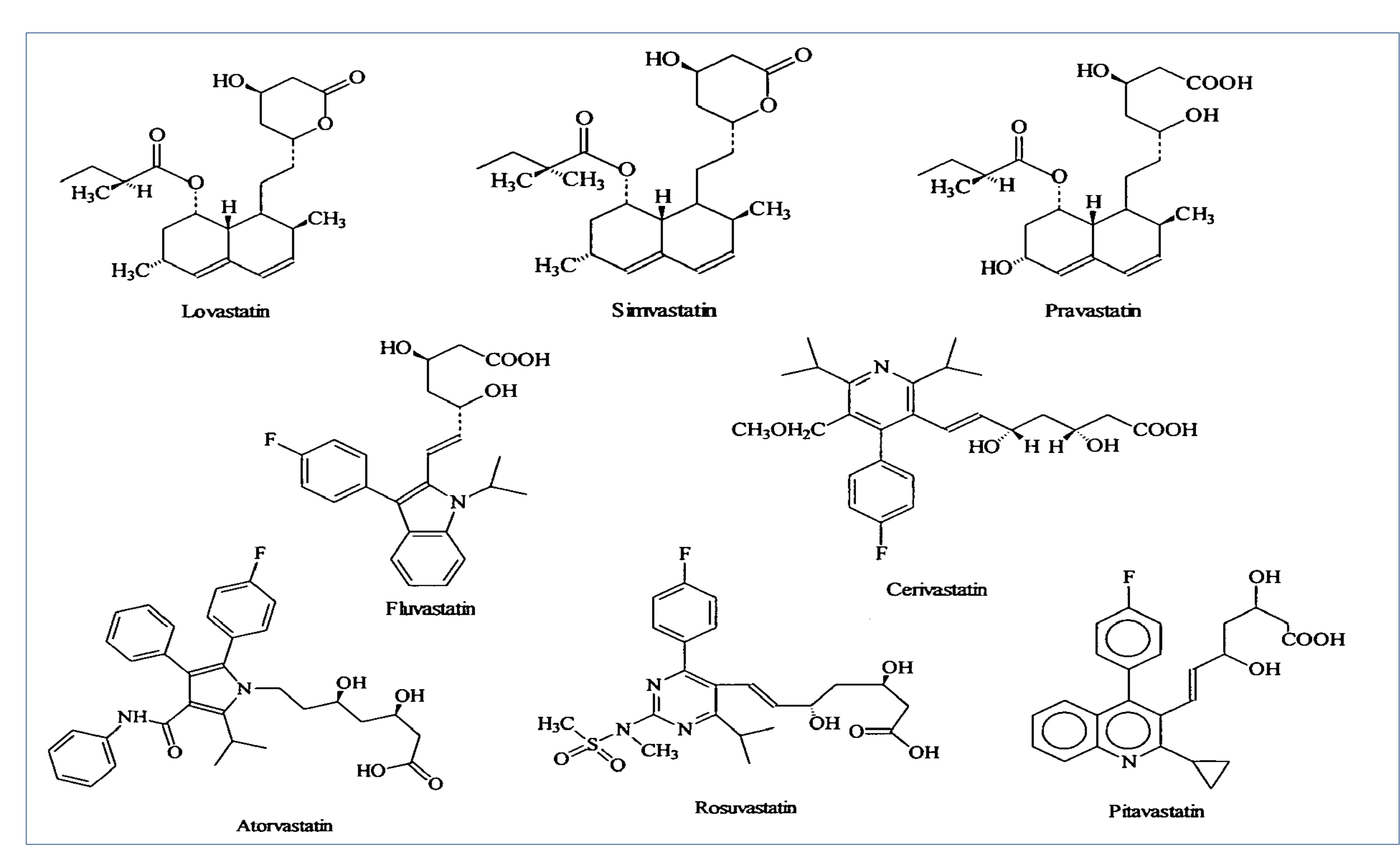

Fig 1. Molecular structure of common statins

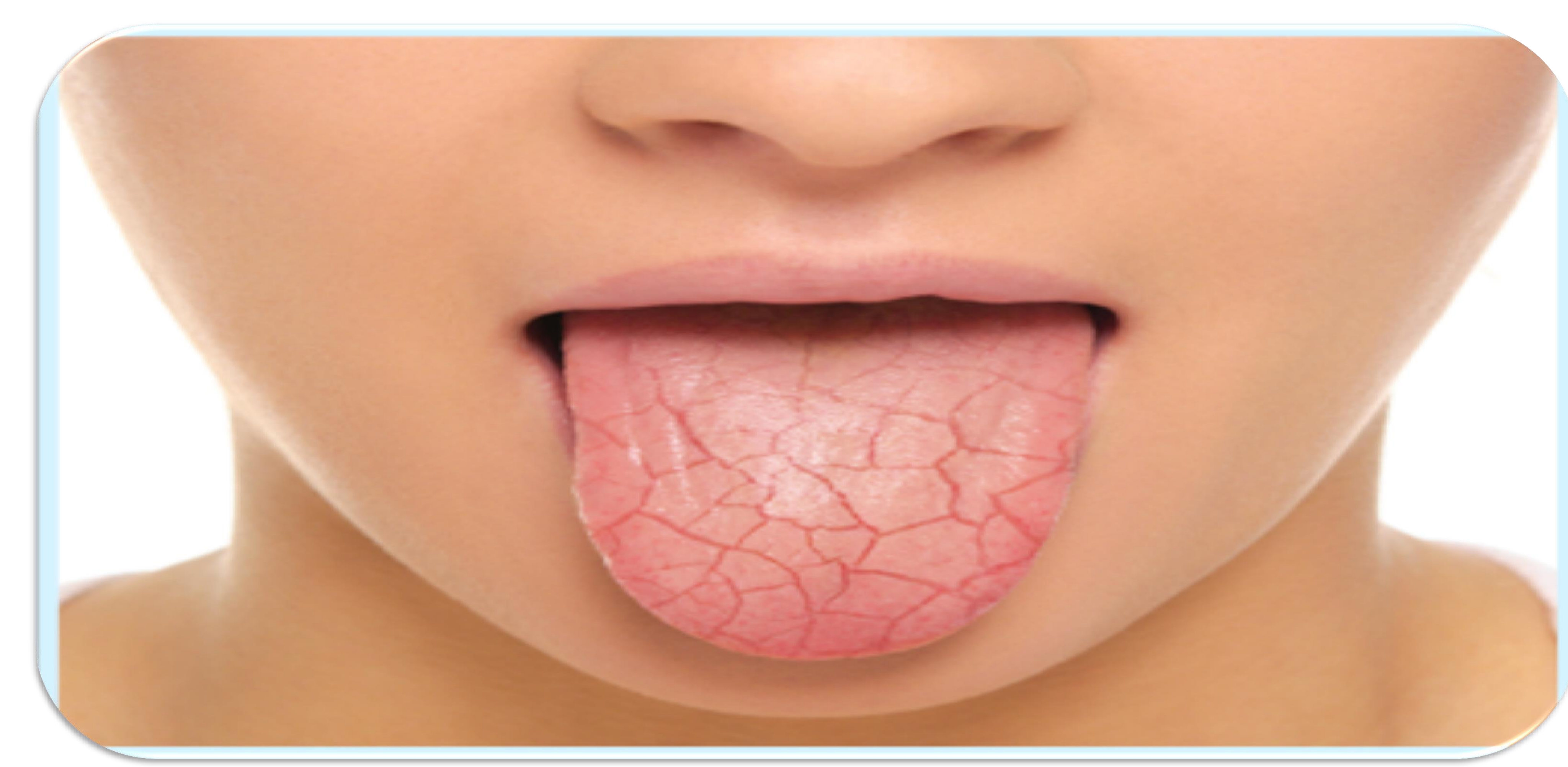

Fig 2. Rough, dry tongue observed in xerostomia

\section{DISCUSSION}

This case demonstrates that statin use can be associated with xerostomia. This is not uncommon as a study in 2010 by Habbab et al found that $75 / 531$ patients $(14.1 \%)$ had xerostomia as a side effect of cardiovascular medications including statins. ${ }^{1}$ This was the most common side effect experienced by patients.

As demonstrated in this case a drug free period minimizes xerostomia. A study by Pascual and colleagues showed that $23 / 26$ (88.5\%) of patients had oral dryness with treatment with statins ${ }^{3}$. Out of these patients 17/23 showed improvements following the discontinuation of statin treatment for a period of two weeks.

Xerostomia is a debilitating side effect of statins. More studies are required to investigate whether patients discontinue this vital medication due to oral side effects such as Xerostomia.

\section{CONCLUSIONS}

Unfortunately side effects such as xerostomia remain unreported by patients owing to their perception as a minor side effect. This indicates that studies underestimate its true prevalence in statin treatment therefore more studies are required on the association between statins and xerostomia.

Xerostomia has long been recognised to a be a difficult symptom to treat, hence multidisciplinary approach is required in its prevention. ${ }^{4}$

\section{REFERENCES}

Habbab KM, Moles DR, Porter SR. Potential oral manifestations of cardiovascular drugs. Oral Dis 2010; 16: 769-73.

'Guggenheimer J, Moore P. Xerostomia: etiology, recognition and treatment. J Am Dent Assoc 2003; 134(1): 61-69.

E, López López J. Adverse side effectives of statins inte JA, Mezquiriz Ferrero X, Borrell Thio 2008; 13(2): E98-101.

Thomson WM. Dry mouth and older people. Aust Dent J 60 2015; ( 1 Suppl):54-63.

CORRESPONDING AUTHOR

Dr Shemin S. Vyas; Email: drsheminvyas@gmail.com 